\title{
Lung cancer screening white paper: a slippery step forward?
}

\author{
To the Editor:
}

We have read with great interest the White Paper on lung cancer screening published jointly by the European Respiratory Society and the European Society of Radiology [1]. While it has some thoughtful insights, it also has some misconceptions.

1) Use of evidence. The benefit of lung cancer screening is controversial. Even in the USA, the limits that Medicare put on screening are very substantial in terms of requiring registries and limiting screening centres. There are different recommendations from scientific societies based on the same evidence (age range, ex-smokers inclusion). The American Academy of Family Physicians and others do not even recommend screening $[2,3]$. The mortality benefit of lung cancer screening is not $20 \%$ but rather $16 \%$ (20\% lower) according to published data using a longer National Lung Screening Trial (NLST) follow-up [4]. This is an important point that reduces the screening benefit. An extremely relevant limitation of lung cancer screening observed in the NLST is a low downstaging. Downstaging is the core objective of any screening programme as it allows the modification of the clinical course of a disease. The first and second incidence rounds of NLST showed $59 \%$ and $64 \%$ of stage I lung cancers compared with $54.6 \%$ in the prevalence round [5]. Stage I downstaging with lung cancer is below $10 \%$. The authors state that $60-80 \%$ of screening-detected cancers will be stage $\mathrm{I}$ and this is an overstatement which can lead to misinterpretation [1]. 30\% of screen-detected lung cancers in incidence rounds are stages III or IV in the NLST.

2) Overdiagnosis. Overdiagnosis exists and is higher than the $18 \%$ observed by Patz et al. [6] when comparing computed tomography (CT) with chest radiography in the NLST. As the White Paper correctly points out, radiography used for lung cancer screening also showed overdiagnosis in other studies [7]. It is logical therefore to think that overdiagnosis is even higher when comparing chest CT with standard practice without screening. Furthermore, overdiagnosis, by definition, biases results towards a better survival. The higher the rate of overdiagnosis in a given screening programme, the higher the benefit achieved (lower mortality and lower adverse effects related to screening).

3) Radiation induced cancers. In our opinion, the White Paper underscores the importance of radiation induced cancers. A person undergoing lung cancer screening would suffer more than 25 low-dose CTs. Considering the number of false positives, even in case of using volumetric criteria to reduce false positives, it is expected that at least one in five incidence screens would be a false positive and therefore need extra image-based work-up. A diagnostic CT uses much more than 2-3 mSv and such screening would occur at least five times in the 25 years of screening. This radiation dose would be higher than that received by atomic bomb survivors or nuclear plant workers $(40 \mathrm{mSv})$ [8].

4) Cost-effectiveness of health interventions. The cost matters, and lung cancer screening which includes diagnostic work-up of large numbers of positive patients is extremely expensive. $\$ 81000$ per quality-adjusted life year (QALY) is a high cost [9], and the upper limit of this estimation is $\$ 186000$. Cost per QALY for ex-smokers, which comprised $52 \%$ of NLST patients, is $\$ 615000$. We have to add the cost of implementation of a lung cancer screening programme, which is also very high (purchase of dedicated CTs, dedicated personnel, an information system and so on). The cost per QALY for tobacco cessation interventions ranges between $€ 1000$ and $€ 5000$ [10]. It is obvious that the savings obtained by quitting smoking are significant. If we consider the cost-opportunity, smoking cessation reduces the risk of many diseases and extends lifespan an average of 10 years [11]. The cost of lung cancer screening might also have important differences depending on the European country applying it. Cost-effectiveness of smoking cessation has to be included in lung cancer screening debate.

It seems too early to promote lung cancer screening in Europe due to the aforementioned reasons. While lung cancer screening was shown as beneficial in only one study, this benefit is not exempt of risks. The downstaging observed is discrete. It is estimated that lung cancer screening might prevent between 6500 and 8000 annual lung cancer deaths in the USA, 5\% of the total annual lung cancer deaths [12]. For each 
1000 screened individuals, lung cancer deaths will be reduced from 21 to 18 (16\%). From different points of view, the impact of screening on lung cancer deaths is very low [13].

Finally, no European study has indicated any benefit related with lung cancer screening and researchers are currently working on a European Lung Cancer pooling study. Recent results of the DANTE (Detection and Screening of Early Lung Cancer with Novel Imaging Technology and Molecular Assays) study do not show any benefit [14], the same as the Danish Lung Cancer Screening Trial [15]. The reason for divergence between European studies and NSLT is currently unknown. It might be due to selection bias in NSLT (younger age or higher education of participants) or the selection of excellent participating centres (low surgical mortality). One last reason, but not less important, is the different health system model used in each geographical area. Is there such strong evidence to promote the introduction of lung cancer screening in Europe? Has its cost-effectiveness been demonstrated compared with cheaper alternatives? Are we in such a hurry that we cannot wait some months for the European Pooling or Nelson study results? With the current evidence, lung cancer screening should be restricted to the individual discussion patient-clinician. In our opinion, the white paper is a slippery step forward that might not be beneficial to European patients and that threatens the economic sustainability of European health services.

- @ERSpublications

The recent white paper on lung cancer has some insights, but also some misconceptions http://ow.ly/RZLmM

Alberto Ruano-Ravina ${ }^{1,2}$, Mariano Provencio-Pulla ${ }^{3,4}$ and Alberto Fernández-Villar

${ }^{1}$ Department of Preventive Medicine and Public Health, University of Santiago de Compostela, Santiago de Compostela, Spain. ${ }^{2}$ CIBER de Epidemiología y Salud Pública, CIBERESP, Spain. ${ }^{3}$ Service of Oncology, Hospital Puerta de Hierro, Madrid, Spain. ${ }^{4}$ Instituto de Investigación Puerta de Hierro, Madrid, Spain. ${ }^{5}$ Service of Neumology, University Hospital Complex of Vigo, Vigo, Spain.

Correspondence: A Ruano-Ravina, Department of Preventive Medicine and Public Health, School of Medicine. C/ San Francisco s/n, University of Santiago de Compostela, 15782, Santiago de Compostela, Spain.

E-mail: alberto.ruano@usc.es

Received: May 282015 | Accepted after revision: June 172015

Conflict of interest: None declared

\section{References}

1 Kauczor H-U, Bonomo L, Gaga M, et al. ESR/ERS white paper on lung cancer screening. Eur Respir J 2015; 46: $28-39$.

2 American Academy of Family Physicians. www.aafp.org/patient-care/clinical-recommendations/all/lung-cancer. html Date last accessed: June 16, 2015.

3 Frauenfelder T, Puhan MA, Lazor R, et al. Early detection of lung cancer: a statement from an expert panel of the Swiss university hospitals on lung cancer screening. Respir Int Rev Thorac Dis 2014; 87: 254-264.

4 Pinsky PF, Church TR, Izmirlian G, et al. The National Lung Screening Trial: results stratified by demographics, smoking history, and lung cancer histology. Cancer 2013; 119: 3976-3983.

5 National Lung Screening Trial Research Team, Aberle DR, Adams AM, Berg CD, et al. Reduced lung-cancer mortality with low-dose computed tomographic screening. N Engl J Med 2011; 365: 395-409.

6 Patz EF Jr, Pinsky P, Gatsonis C, et al. Overdiagnosis in low-dose computed tomography screening for lung cancer. JAMA Intern Med 2014; 174: 269-274.

7 Marcus PM, Bergstralh EJ, Zweig MH, et al. Extended lung cancer incidence follow-up in the Mayo Lung Project and overdiagnosis. J Natl Cancer Inst 2006; 98: 748-756.

8 McCunney RJ, Li J. Radiation risks in lung cancer screening programs: a comparison with nuclear industry workers and atomic bomb survivors. Chest 2014; 145: 618-624.

9 Black WC, Keeler EB, Soneji SS. Cost-effectiveness of CT screening in the National Lung Screening Trial. N Engl J Med 2015; 372: 388.

10 Flack S, Taylor M, Trueman P. Cost-Effectiveness of Interventions for Smoking Cessation. University of York; 2007 p. 77. Available from www.nice.org.uk/guidance/ph10/resources/smoking-cessation-services-economics-modellingreport-2 Date last accessed: June 16, 2015.

11 Jha P, Peto R. Global effects of smoking, of quitting, and of taxing tobacco. N Engl J Med 2014; 370: 60-68.

12 Harris RP. Starting a new discussion about screening for lung cancer. JAMA 2015; 313: 717-718.

13 Woolf SH, Purnell JQ, Simon SM, et al. Translating evidence into population health improvement: strategies and barriers. Annu Rev Public Health 2015; 36: 463-482.

14 Infante M, Cavuto S, Lutman FR, et al. Long-Term Follow-up Results of the DANTE Trial, a Randomized Study of Lung Cancer Screening with Spiral Computed Tomography. Am J Respir Crit Care Med 2015; 191: 1166-1175.

15 Saghir Z, Dirksen A, Ashraf H, et al. CT screening for lung cancer brings forward early disease. The randomised Danish Lung Cancer Screening Trial: status after five annual screening rounds with low-dose CT. Thorax 2012; 67: 296-301. 